\title{
Rf-induced transport of Cooper pairs in superconducting single electron transistors in a dissipative environment
}

\author{
S. V. Lotkhov A S. A. Bogoslovsky, A. B. Zorin, and J. Niemeyer \\ Physikalisch-Technische Bundesanstalt, Bundesallee 100, 38116 Braunschweig, Germany
}

(Dated: November 15, 2018)

\begin{abstract}
We investigate low-temperature and low-voltage-bias charge transport in a superconducting $\mathrm{Al}$ single electron transistor in a dissipating environment, realized as on-chip high-ohmic Cr microstrips. In our samples with relatively large charging energy values $E_{\mathrm{c}}>E_{\mathrm{J}}$, where $E_{\mathrm{J}}$ is the energy of the Josephson coupling, two transport mechanisms were found to be dominating, both based on discrete tunneling of individual Cooper pairs: Depending on the gate voltage $V_{\mathrm{g}}$, either sequential tunneling of pairs via the transistor island (in the open state of the transistor around the points $Q_{\mathrm{g}} \equiv C_{\mathrm{g}} V_{\mathrm{g}}=e \bmod (2 e)$, where $C_{\mathrm{g}}$ is the gate capacitance) or their cotunneling through the transistor (for $Q_{\mathrm{g}}$ away of these points) was found to prevail in the net current. As the open states of our transistors had been found to be unstable with respect to quasiparticle poisoning, highfrequency gate cycling (at $f \sim 1 \mathrm{MHz}$ ) was applied to study the sequential tunneling mechanism. A simple model based on the master equation was found to be in a good agreement with the experimental data.
\end{abstract}

\section{INTRODUCTION}

During the last few years, the ultrasmall Josephson junctions have aroused the interest of researchers as convenient objects for experimental investigations of macroscopic quantum effects. A variety of their unique features formed the basis for possible applications in quantum information processing (see, e.g., review 1] and references therein), Cooper pair electrometry 22, and metrology (accurate current sources on the basis of Cooper pair pumps [3]).

The Cooper pair dynamics in such junctions appears as a result of the competition between Coulomb and Josephson effects (see, e.g., 4|), and it is strongly affected by interaction with the electrodynamic environment (for the review, see [5]). In particular, the Cooper-pair transport is very sensitive to dissipative properties of the environment. Quantitatively, these properties are characterized by the real part of the frequency-dependent impedance of the environment $\operatorname{Re} Z(\omega)$.

In the low-impedance case, $|Z(\omega)| \ll R_{\mathrm{Q}}$, where $R_{\mathrm{Q}} \equiv$ $h / 4 e^{2} \approx 6.45 \mathrm{k} \Omega$ is the resistance quantum, the tunneling of Cooper pairs is elastic and occurs at zero bias voltage as a coherent process. The resulting net current presents the supercurrent as in the "classical" Josephson junction [6], because the Josephson phase across the junction is well defined. In most of the experimental systems, small but finite dissipation of the measuring circuitry $|Z(\omega)| \lesssim R_{\mathrm{Q}}$ causes phase fluctuations which transform the zero voltage supercurrent into a supercurrent peak of finite width located at small voltages (see Ref. 7] and the references therein). In contrast to the former two cases, tunneling of pairs in the junction embedded in a rather dissipative environment, $\operatorname{Re} Z(\omega) \gtrsim R_{\mathrm{Q}}$, is related to an extensive energy exchange with the environment. Tunneling of pairs in this case occurs incoherently and can be considered in terms of individual events. The first ob- servation of dissipative tunneling of single Cooper pairs was reported by Kuzmin et al. [8] for a simple system of a small single junction connected in series to a compact high-ohmic resistor.

Several different transport mechanisms were reported for a two-junction superconducting device (single electron transistor, SET) supplied by the high-ohmic resistor (see Fig. 11). One of the mechanisms - sequential tunneling of pairs (ST) through either junction - was studied theoretically by Wilhelm et al. 9], and experimentally by Kycia et al. 10] and by Lu et al. 11]. These experiments were performed in an Al SET positioned on top of a two-dimensional electron gas with locally tunable dissipation (ground plane conductance). Another mechanism based on a higher-order process of simultaneous tunneling of pairs in both junctions - i.e., the cotunneling of pairs (CT) - was recently studied in detail [12]. Moreover, resonant tunneling of single Cooper pairs (RT) 13. was found to be responsible for the charge transport at somewhat larger bias voltages $\sim 2 E_{\mathrm{c}} / e<2 \Delta_{\mathrm{Al}}$ [14]. Here, $E_{\mathrm{c}} \equiv \frac{e^{2}}{2 C_{\Sigma}}$ is the charging energy, $C_{\Sigma}=2 C_{\mathrm{T}}+C_{\mathrm{g}}$ the total capacitance of the transistor island, $C_{\mathrm{T}}$ the tunnel capacitance (in a symmetric device), $C_{\mathrm{g}}$ the gate capacitance, and $\Delta_{\mathrm{Al}} \approx 200 \mu \mathrm{eV}$ the superconducting energy gap of Al. The RT process involves resonant mixing of charge states across one junction, completed by tunneling of Cooper pairs in the second junction with energy dissipation in the resistor.

In our recent paper 12] we had shown that at low temperatures and small voltage bias, $\mathrm{CT}$ dominates in the net current for gate charges $Q_{\mathrm{g}} \equiv C_{\mathrm{g}} V_{\mathrm{g}},\left|Q_{\mathrm{g}}\right| \lesssim e / 2$, where ST is blocked due to the Coulomb barrier $\gtrsim 2 E_{\mathrm{c}}$, provided the Josephson coupling energy in the junctions $E_{\mathrm{J}}$ is small, $E_{\mathrm{J}} \ll E_{\mathrm{c}}$. The $\mathrm{ST}$ process becomes energetically favourable in the points around $Q_{\mathrm{g}} \approx e \bmod (2 e)$, giving rise to the "open" states of the SET. In the experiment, these states are often unstable towards spon- 


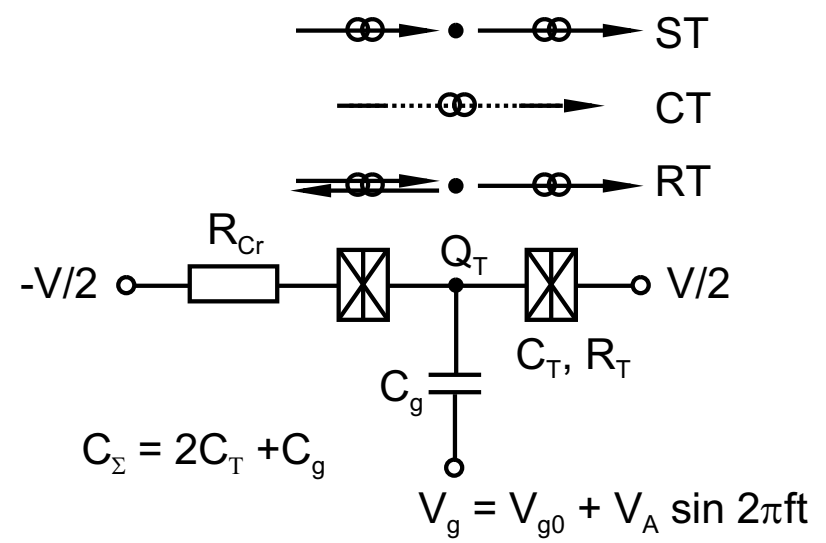

FIG. 1: Equivalent circuit diagram of a symmetric superconducting SET and different mechanisms of Cooper pair transport: sequential tunneling (ST), cotunneling (CT), and resonant tunneling (RT).

taneous tunneling of non-equilibrium quasiparticles (the so-called, quasiparticle poisoning effect, see, e.g., 15] and [16]). Study of the crossover between ST and CT is therefore a challenging task.

In this paper, besides the observation of the CT current, we succeeded in realizing of the regime, where the $\mathrm{ST}$ process dominates over CT. We focus on the range of small bias voltages $e V \ll 2 \Delta_{\mathrm{Al}}$, where tunneling of Cooper pairs dominates over that of quasiparticles, and RT is energetically unfavourable 13. In order to circumvent quasiparticle poisoning of the island in the open states, we applied a high-frequency signal of sufficient amplitude to the gate and measured the time-averaged $I-V$ dependencies. The measured curves were compared with the results provided by a simple model based on the master equation approach. We considered the current evolution along the gate cycle. As expected, it was characterized by the major input from ST in the open states. Our calculation also showed that the contribution of ST current, averaged over the whole cycle, dominates over that of CT.

\section{THEORETICAL BACKGROUND}

At voltages $e V \ll 2 \Delta_{\mathrm{Al}} \approx 400 \mu \mathrm{eV}$, which are well below the thresholds for the different processes incorporating tunneling of quasiparticles (see, e.g., Josephsonquasiparticle cycles in [13|, 17 | and [18]), the tunneling of quasiparticles is rare [16], 19], and the net current is related to the transport of Cooper pairs.

The Cooper pair dynamics in a superconducting SET with $E_{\mathrm{J}} \ll E_{\mathrm{c}}$ and appreciable dissipation is qualitatively similar to that of electrons in the normal-state SET described by the orthodox theory [20]. In particular, a quantum state of the whole system can be described by the number $n$ of extra Cooper pairs on the island of the SET. The Josephson coupling in the junctions can be regarded as a small perturbation in the total Hamiltonian of the system, giving rise to random tunneling events of different orders [5].

Since tunneling of Cooper pairs does not produce excitations, it is dissipating properties of the environment that define the functional dependence of a particular tunneling rate on the electrostatic energy difference $E$ associated with the tunneling event. The corresponding probability function $P(E)$ characterizes the ability of the environment to exchange an energy $E$ with the tunneling pair. For a given temperature, the shape of the $P$ function depends on the environmental impedance $Z(\omega)$ as well as on the tunnel capacitances of the junctions. In some important cases, $P(E)$ can be expressed analytically (see, for example, below).

The process of the lowest order of perturbation is known as direct tunneling of a pair in either junction. It changes the charge state of the SET island by $\pm 2 e$. A rate of direct tunneling can be expressed as 21]:

$$
\Gamma(E)=\frac{\pi}{2 \hbar} E_{J}^{2} P(E)
$$

The validity range of the perturbative approach Eq. (II) strongly depends on the dissipative properties of the environment and is generally restricted to SETs with relatively weak Josephson coupling and/or large damping, i.e., when:

$$
E_{\mathrm{J}} P(E) \ll 1 \text {. }
$$

An analysis performed in Ref. [5] shows that in the case of zero temperature and a low-ohmic environment, $\operatorname{Re} Z(\omega) \ll R_{\mathrm{Q}}$, the probability $P(E)$ grows infinitely around zero energies, and the condition Eq. (2) is always violated at small voltages. On the contrary, at finite experimental temperatures $T \sim 100 \mathrm{mK}$ and/or in the case of moderate dissipation, $\operatorname{Re} Z(\omega) \sim R_{\mathrm{Q}}$, the function $P(E) \sim 4 E_{\mathrm{c}}^{-1}$ is distributed over the broad range of energies so that the conditional equation (2) can be reformulated as $\lambda \equiv E_{\mathrm{J}} / E_{\mathrm{c}} \ll 4$. For the junctions in a high-ohmic environment, $\operatorname{Re} Z(\omega)>R_{\mathrm{Q}}$, the conditional equation (2) is even less restrictive at low energies, where $P$ is a power function of $E$ (see Eq. (111) in Ref. $[5]$ ). As a result, in the systems with noticeable dissipation, the approximation of discrete tunneling events can even be applied to junctions with relatively strong Josephson coupling.

For small energies, the external impedance can be considered to be frequency-independent $Z(\omega)=Z(0)$ (purely ohmic) and one obtains an analytic expression 22] for the $P$-function for tunneling in individual junc- 
tions of a symmetric transistor:

$$
\begin{aligned}
P(E) & =\frac{z^{2 z}}{8 E_{c}}\left(\frac{2 E_{c}}{\pi^{2} k_{B} T}\right)^{1-2 z} e^{-2 \gamma z} \\
& \times \frac{\left|\Gamma\left[z-j\left(E / 2 \pi k_{B} T\right)\right]\right|^{2}}{\Gamma(2 z)} e^{E / 2 k_{B} T},
\end{aligned}
$$

which is valid for low temperatures and energies $E \ll$ $\hbar \omega_{Z}$. The value of the threshold frequency $\omega_{Z}$ of the ohmic approximation is defined for each experimental layout by all environmental components (including resistor, tunnel and stray capacitances and inductances according to the chip layout). In our experiment, the typical value was evaluated $\omega_{Z} \sim 4 \times 10^{10} \mathrm{~s}^{-1}\left(\hbar \omega_{Z} \sim 25 \mu \mathrm{eV}\right.$, cf. experimental section of this paper). In Eq. (3), $\Gamma$ is a gamma function and $\gamma \approx 0.577$ Euler's constant. The dimensionless resistance $z=\frac{\rho}{4}$, where $\rho \equiv Z(0) / R_{\mathrm{Q}}$, accounts for the factor $1 / 4$ (in comparison to the case of a single junction or cotunneling) which arises from the partial decoupling effect of the tunnel junction from the environment due to the other junction (cf. network considerations in Ref. [5]). The low-temperature approximation requires $k_{\mathrm{B}} T \ll 4 E_{\mathrm{c}} / \pi z$ which in most of the cases is valid in the whole $\mathrm{mK}$ temperature range.

For tunneling in the left (right) junction of the symmetric SET, the energy difference is:

$$
E(V, Q)=e V-4 E_{\mathrm{c}}\left(1 \pm \frac{Q}{e}\right) ;
$$

it depends on voltage bias $V$ and effective charge $Q=Q_{\mathrm{g}}$ $+Q_{\mathrm{T}}$, where $Q_{\mathrm{T}}=2 n e+m e$ is a charge of $n$ extra Cooper pairs and $m$ extra quasiparticles on the island. Similar to the case of a normal-state SET (see, e. g., Ref. [20]), the energy relations in Eq. (4) can be mapped in the $V-V_{\mathrm{g}}$ plane, giving a 2e-periodic diamond-shaped stability diagram for Cooper pairs. As shown in Fig. [2(a), this diagram is featured by a blockade domains with the stable number of extra pairs on the island, as well as by the regions of open states, where at least two alternating charge states are available for realization of sequential transport of Cooper pairs through the SET. At small biases, the values of the gate charge for the open states of the SET, e.g., $Q_{\mathrm{g}} \approx e \bmod (2 e)$ for $m=0$, correspond to a small vicinity of the resonance points $Q= \pm e$.

Similar to the normal-state SET, it is also possible to calculate the net ST current at low temperatures, using the master equation approach. In particular, in the important case of a small voltage bias, the stationary population of the island is dominated by two charge states, $n=0,1$, with the occupation probabilities $\sigma_{n}=\sigma_{n}\left(v, v_{\mathrm{g}}\right)$ which can be found with the help of the detailed balance principle:

$$
\begin{gathered}
\sigma_{0}=\frac{\Gamma_{1}^{-}(1)+\Gamma_{2}^{+}(1)}{\Gamma_{\Sigma}}, \\
\sigma_{1}=\frac{\Gamma_{1}^{+}(0)+\Gamma_{2}^{-}(0)}{\Gamma_{\Sigma}},
\end{gathered}
$$

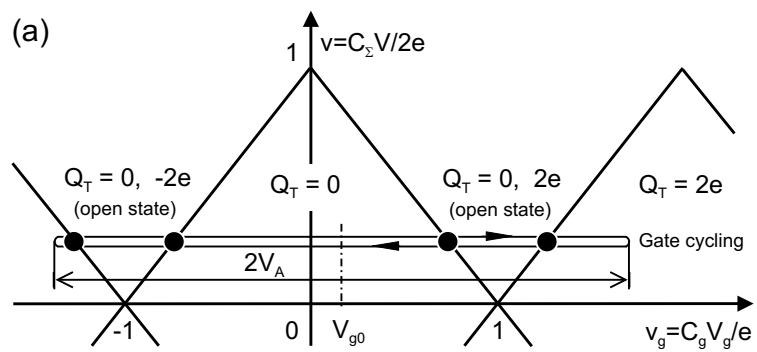

(b)
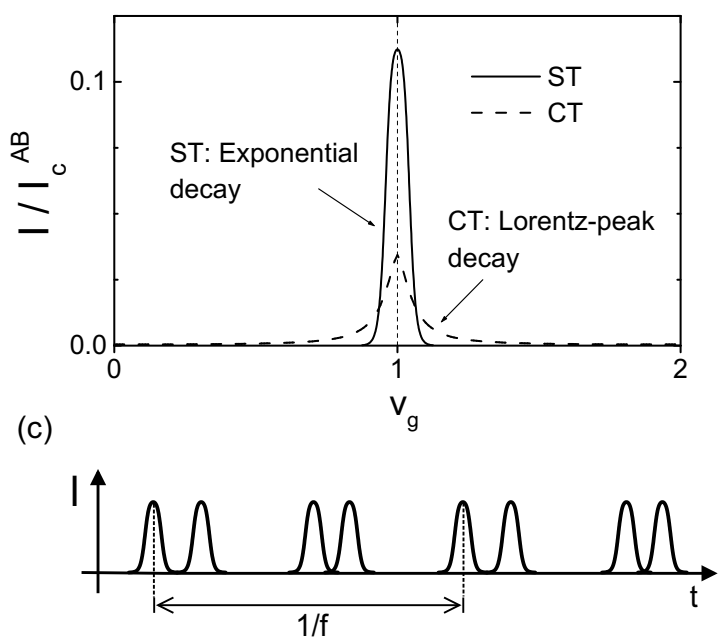

FIG. 2: (a) Stability diagram for Cooper pairs in a singlecharge transistor for $m=0$. The closed circles show the points of Cooper pair resonances in individual junctions, $E=0$. The cycling amplitude $V_{\mathrm{A}}$ is chosen to sweep over two open domains. (b) ST and CT currents, normalized to the Ambegaokar-Baratoff value $I_{c}^{\mathrm{AB}}$, were calculated as a function of the gate charge for the parameters of sample 2 (see Table and fixed $V=25 \mu \mathrm{V}$ and $T=100 \mathrm{mK}$. (c) Schematic representation of an rf-induced periodic sequence of current pulses resulting from cycling over two open domains (as shown in (a)). The amplitude of pulses given by the number of tunneling pairs is fluctuating.

where $\Gamma_{\Sigma}=\Gamma_{1}^{-}(1)+\Gamma_{1}^{+}(0)+\Gamma_{2}^{+}(1)+\Gamma_{2}^{-}(0)$, and $\Gamma_{1,2}^{ \pm}(n)=$ $\Gamma_{1,2}^{ \pm}\left(v, v_{\mathrm{g}}, Q_{\mathrm{T}}\right)$ denote the rates in the left (right) junction in the positive (negative) direction and can be calculated with the help of Eqs. (11), (3) and (4). The net current is obtained as a sum over the states $n$ :

$$
\begin{aligned}
I & =-2 e\left[\sigma_{0} \Gamma_{1}^{+}(0)-\sigma_{1} \Gamma_{1}^{-}(1)\right] \\
& =-2 e\left[\sigma_{1} \Gamma_{2}^{+}(1)-\sigma_{0} \Gamma_{2}^{-}(0)\right] .
\end{aligned}
$$

An example of the ST current dependence on the gate voltages $I\left(v, v_{\mathrm{g}}\right)$, calculated for fixed bias voltage and the parameters of one of our samples, is shown in Fig. 2(b). The net current, $I \sim 0.1 I_{\mathrm{c}}^{\mathrm{AB}} \sim 1 \mathrm{nA}$, where $I_{\mathrm{c}}^{\mathrm{AB}}$ is the Ambegaokar-Baratoff critical current, is significant in the open domain between the resonant bound- 
aries and decays exponentially with the height of the barrier $|E|$, see Eq. (4), inside the blockade domains: $I \propto \exp \left(-|E| / 2 k_{\mathrm{B}} T\right)$.

Simple analysis of the time-dependent master equation shows that the time $\tau$ the system takes to reach its stationary state, when moving between the operating points in the open domain (where the current is considerable), $\tau \sim \Gamma_{\Sigma}^{-1} \sim e / 2 I \sim 0.1 \mathrm{~ns}$, is well below the experimental range of time $\sim 10$ ns of passing one open domain when the gate is cycled at $f \sim 1 \mathrm{MHz}$. Making use of this fact, the quasi-stationary master-equation approach can be applied and the stationary ST currents considered for each point of the cycling trajectory, even when modeling high-frequency behaviour of the system. The gate cycling in particular will result in a regular pattern of ST current pulses, for example, 4 pulses per cycle which involves 4 open domains (see Fig. 2(c)), and the mean ST current, $\bar{I}_{\text {st }}$, can be obtained by averaging the values of $I\left(v, v_{\mathrm{g}}\right)$ over the cycling period.

A contribution $I_{\mathrm{ct}}$ to the total net current due to higher-order processes, i.e., cotunneling of Cooper pairs, can be analyzed using the lines developed in Ref. 12]. It was shown in particular that an SET can be modeled by a single junction with an effective, gate-voltage-dependent, Josephson coupling Hamiltonian $H_{\mathrm{tr}}(\phi, Q)$, where $\phi$ is the overall Josephson phase. The coupling is $2 \pi$-periodic in $\phi$, but its shape is generally different from $-\cos \phi$. Expansion of the coupling Hamiltonian into Fourier series (see Eq. (4) in Ref. 12]) makes it possible to assess the individual contributions to the $\mathrm{CT}$-current from the different-order cotunneling channels, each one simultaneously transporting a certain number $k$ of Cooper pairs through the SET. Similar to direct tunneling, the rate formula in Eq. (1) can be used to calculate the partial cotunneling rates due to each channel, with the substitution $E=2 k e V$, taking account of cotunneling of $k$ pairs. The effective value of Josephson coupling energy for each channel is given by the corresponding Fourier coefficient $E_{\mathrm{J}}^{(k)}(Q)$, and the dimensionless resistance $k^{2} \rho$ must be used in Eq. (3) for the $P$-function.

It was shown in Ref. 12] that the lowest-order, i.e. single-pair, CT-process with $k=1$ generally dominates in the CT-current through the SET, and the principal Fourier term can provide a reasonable approximation for the coupling Hamiltonian: $H_{\mathrm{tr}}(\phi, Q) \approx-E_{\mathrm{J}}^{1}(Q) \cos (\phi)$. It can further be demonstrated that dropping-out of the higher terms causes an error of $\delta I_{\mathrm{ct}} \sim 10 \%$ at most which is obtained, in a symmetric SET, in the degeneracy points $Q \approx \pm e$ (see Eq. 6 in Ref. [12]). Around these points, the CT-current almost behaves like a Lorentzian peak, and at $Q \rightarrow 0$ it decays more slowly than the exponentially suppressed ST current (see Fig. 2(b)). As a result, the CT-current dominates in the net current inside the blockade domains.

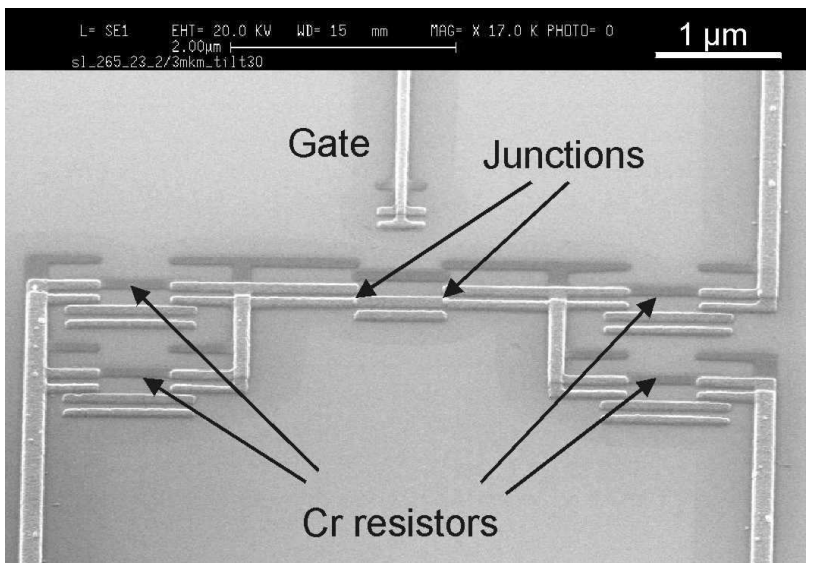

FIG. 3: SEM-micrograph of the $\mathrm{Al} / \mathrm{AlO}_{\mathrm{x}} / \mathrm{Al}-\mathrm{Cr}$ - transistor. The configuration with two parallel $\mathrm{Cr}$ microstrips on each side was implemented for the purpose of directly measuring of $R_{\mathrm{Cr}}$ as half the resistance between both right-hand arms of the device.

\section{EXPERIMENTAL SETUP}

The samples were fabricated by the shadow evaporation technique. The details of our fabrication process can be found in [23]. Each structure was built on three almost congruent metal layers (see Fig. 3) deposited at different angles through the same pattern in the mask with hanging bridges. The layers were of $\mathrm{Cr}, 7 \mathrm{~nm}$ thick and $0.1 \mu \mathrm{m}$ wide as used for high-ohmic resistors, and two layers of $\mathrm{Al}, 30$ and $35 \mathrm{~nm}$ in thickness, evaporated with intermediate oxidation of the first layer to form the tunnel barriers. The nominal tunnel junction area was $40 \mathrm{~nm} \times 80 \mathrm{~nm}$.

The results reported in this paper were obtained on two SETs (samples 2 and 3, see Table I) in a dissipating environment, realized as $\mathrm{Cr}$ microresistors with $Z(0)=R_{\mathrm{Cr}} \approx 3$ and $12 \mathrm{k} \Omega$ as well as on a reference sample 1 without resistor, i.e., $R_{\mathrm{Cr}}=0$. The corresponding values of the dimensionless resistance parameter were $\rho \approx 0.5,2$ and 0 . Due to the linearity and small lengths of the resistors it was possible to consider them as lumped elements (ohmic resistors) and neglect their distributed self-capacitance $(\approx 65 \mathrm{aF} / \mu \mathrm{m}$, as earlier estimated for the similar layout [24]).

Ambegaokar-Baratoff critical currents of individual junctions were estimated, assuming symmetry of the transistors: $I_{c}^{\mathrm{AB}}=\frac{\pi \Delta_{\mathrm{Al}}}{2 e R_{\mathrm{T}}} \approx 13-15 \mathrm{nA}$, where $R_{\mathrm{T}}$ is the tunnel resistance. The corresponding values of the Josephson energy were $E_{\mathrm{J}}=\frac{\Phi_{0}}{2 \pi} I_{\mathrm{c}}^{\mathrm{AB}} \approx 26-32 \mu \mathrm{eV}$, where $\Phi_{0}=\frac{h}{2 e}$ is the flux quantum. Roughly evaluated with the help of a similar device with $R_{\mathrm{Cr}}=0$ in the normal state, the characteristic charging energy of the transistor island was $E_{\mathrm{c}} \approx 150$ to $170 \mu \mathrm{eV}$. The ratio of the characteristic energies in all our samples, $\lambda \approx 0.15-0.2$, 
TABLE I: Sample parameters.

\begin{tabular}{|c|c|c|c|c|c|c|}
\hline Sample & $R_{\mathrm{T}}(k \Omega)$ & $R_{\mathrm{Cr}}(k \Omega)$ & $I_{\mathrm{c}}^{\mathrm{AB}}(\mathrm{nA})$ & $E_{\mathrm{J}}(\mu \mathrm{eV})$ & $E_{\mathrm{c}}(\mu \mathrm{eV})$ & $E_{\mathrm{J}} / E_{\mathrm{c}}$ \\
\hline 1 & 20 & 0 & 15 & 32 & 150 & 0.22 \\
\hline 2 & 20 & 3 & 15 & 32 & 150 & 0.22 \\
\hline 3 & 25 & 12 & 13 & 26 & 170 & 0.15 \\
\hline
\end{tabular}

indicates that the SET charging energy dominated over the Josephson energy of the junctions. 1e-modulation period in the $I-V_{\mathrm{g}}$ curve without $\mathrm{rf}$ drive was $\approx 20 \mathrm{mV}$ yielding $C_{\mathrm{g}} \approx 8 \mathrm{aF}$.

$I-V$ characteristics were measured at the base temperature of the dilution refrigerator of about $10 \mathrm{mK}$. The bias and gate lines were equipped with microwave filters made of Thermocoax ${ }^{\circledR}$ cables, $1.5 \mathrm{~m}$ in length, each thermally anchored to the mixing chamber. The filters provided reliable electromagnetic isolation of the sample inside the shielded sample holder at frequencies above $f \sim 1 \mathrm{GHz}[25]$.

\section{EXPERIMENTAL RESULTS}

As discussed above, observation of ST current should be possible in the vicinity of the resonance points $Q=$ $\pm e$. The parity effect in a superconducting SET (see, e. g., Refs. [16], 19] and 26]), basically makes it possible to reach these points through appropriately setting the gate voltage, provided quasiparticle poisoning (QPP) effects are absent (the value of $m$ is zero or, at least, fixed), and the experimental gate modulation is 2e-periodical. Unfortunately, the effect of QPP did not allow us to reach the resonance points and hindered the observation of ST currents in the dc regime: The observed periodicity was strictly $1 \mathrm{e}$, corresponding to the range of the island charge $-e / 2<Q<e / 2$, i. e., inside the ST blockade domain at low bias voltages.

The effect of QPP is generally supposed to originate from various experimental non-idealities including subgap quasiparticle states [27], out-of-equilibrium quasiparticles [28], back influence of the SET electrometer on the island (box) 29], etc. It involves uncontrolled excitation of quasiparticles in the outer electrodes and their tunneling into the island of SET, thus changing $m$ and minimizing the charging energy of the system. In particular, in the resonant points $Q_{\mathrm{g}}=e \bmod (2 e)$ and $V=0$, the charging energy is minimized, when one extra quasiparticle enters the island resulting in $Q=0$ and Coulomb blockade of ST. The poisoning rate $\gamma_{\mathrm{B}}$ depends on the number of excitations and the subgap tunnel resistance. Typically $\gamma_{\mathrm{B}} \sim 16-1000 \mathrm{~s}^{-1}$ (see, e.g., Ref. 29]).

In Fig. 4 the dc $I-V$ curves are shown for integer and half-integer values of $Q$ in the system with considerable dissipation $\left(R_{\mathrm{Cr}}=3 \mathrm{k} \Omega\right.$, sample 2$)$ in comparison to those for a similar SET fabricated without engineered dissipation $\left(R_{\mathrm{Cr}}=0\right.$, sample 1$)$. As expected for the block-

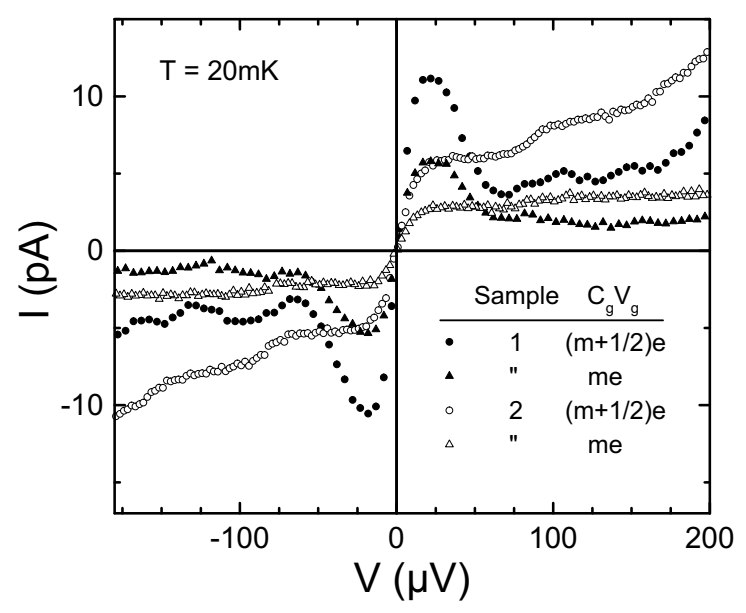

FIG. 4: Dc $I-V$ curves of two SETs with and without onchip resistor, samples 1 and 2 , demonstrate the effects of both gate voltage and dissipative suppression of cotunneling.

ade state, the dc currents were small (up to $\sim 10 \mathrm{pA}$ ) and could be attributed to the higher-order $\mathrm{CT}$ process. In good agreement with the theory [12], the CT-current was modulated by the gate with a maximum-to-minimum ratio of $\approx 2$ within the accessible range of $-e / 2<Q<e / 2$.

To avoid the effect of QPP and reach the open domain for ST at least for a short time, we applied a periodic gate signal of frequency $f \sim 1 \mathrm{MHz}$ which was well above a typical quasiparticle tunneling rate. Due to these fast oscillations, the actual values of $Q$ along the gate sweep were expected to be less affected by the relatively rare quasiparticle tunneling than in the dc mode. As a result, the net current averaged over many cycles should include a substantial contribution due to ST in the open domains.

The $I-V$ curves measured in this regime are shown in Fig. 5 for sample 2 and several rf amplitudes of the gate signal $V_{\mathrm{A}}, 1<C_{\mathrm{g}} V_{\mathrm{A}} / e<2$. The most striking feature of these curves is the dramatic increase in the SET current, demonstrating a large contribution from the open domains involved in gate cycling (cf. scale of rf-induced current in Fig. [5 with that of dc $I-V$ curves of this sample in Fig. (4). At fixed bias, the rf-induced current was a 1e-periodic function of the dc gate shift $V_{g 0}$ (cf. top inset in Fig. 5) which shows that the effect of QPP was still substantial in a long time scale.

A rough evaluation of the contribution of both ST and 


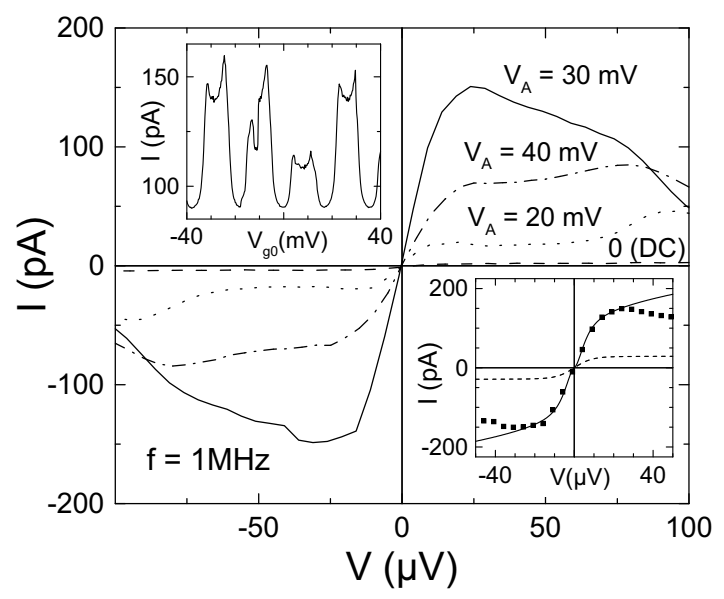

FIG. 5: Rf-induced currents in sample $2\left(R_{\mathrm{Cr}} \approx 3 k \Omega\right)$ for different cycling amplitudes. The cycle was centered in the middle of the blockade domain. Top inset: Rf-induced current vs. dc gate shift $V_{g 0}$ measured at $V=50 \mu \mathrm{V}$ for cycling at $f=1 \mathrm{MHz}$ and a relatively small amplitude $V_{\mathrm{A}}=20 \mathrm{mV}$, i. e., $C_{\mathrm{g}} V_{\mathrm{A}} / e=1$. Bottom inset: $I-V$ curve (symbols) at $V_{\mathrm{A}}$ $=30 \mathrm{mV}\left(C_{\mathrm{g}} V_{\mathrm{A}} / e=1.5\right)$ compared with its fit $\bar{I}_{\text {tot }}(V)$ (solid line) made at fixed $E_{\mathrm{c}}=150 \mu \mathrm{eV}$. The fitting values were: $E_{\mathrm{J}}=40 \mu \mathrm{V}, R_{\mathrm{Cr}}=3.3 k \Omega, C_{\mathrm{g}} V_{\mathrm{A}} / e=1.3, T=100 \mathrm{mK}$. The dashed line shows the calculated contribution of CT, $\bar{I}_{\mathrm{ct}}(V)$.

$\mathrm{CT}$ to the average current in the rf mode can be made with the help of duty-cycle weighting of typical open domain values. For sample 2 , these values were estimated to be $I_{\mathrm{st}} \approx 1.5 \mathrm{nA}$ and $I_{\mathrm{ct}} \approx 0.3 \mathrm{nA}$, respectively. Further assuming $V=25 \mu \mathrm{V}$ and $V_{\mathrm{A}}=30 \mathrm{mV}$, the duty cycle is $\approx 0.05$ producing the estimates: $\bar{I}_{\mathrm{st}} \approx 100 \mathrm{pA}$ and $\bar{I}_{\mathrm{ct}} \approx 20 \mathrm{pA}$, respectively. The total current value $\bar{I}_{\text {tot }}=\bar{I}_{\mathrm{st}}+\bar{I}_{\mathrm{ct}} \approx 120 \mathrm{pA}$ thus obtained agree with the order of magnitude of the experimental data in Fig. 5

For more accurate evaluation of $\bar{I}_{\mathrm{st}}$, we applied the quasi-static master equation approach (Eqs. (5)-(6)) and, for the total current $\bar{I}_{\text {tot }}$, also took the cycle average $\bar{I}_{\mathrm{ct}}$ of CT current into account. We found satisfactory agreement between calculated and experimental $I-V$ curves in the low voltage region, $V \lesssim 25 \mu \mathrm{V}$ (see the bottom inset in Fig. 5). Noticeable discrepancy of the data at higher voltages in our opinion points to the upper limit of the frequency-independent behaviour of the environmental impedance assumed in Eq. (3) for the calculation of the $P$-function. It is still difficult to explain the relatively low value of the cutoff frequency $\omega_{Z}=e V / \hbar \sim 4 \times 10^{10} \mathrm{~s}^{-1}$ for our short $(\sim 0.5 \mu \mathrm{m}-$ long) microstrips (cf. cut-off frequencies $\sim 10^{11} \mathrm{~s}^{-1}$ for $\operatorname{Re} Z(\omega)$ of similar microstrips in Ref. 24]).

The height of the current peaks was found to quasiperiodically depend on the drive amplitude, with a tendency to saturate at higher amplitudes (cf. Fig. [6] show-

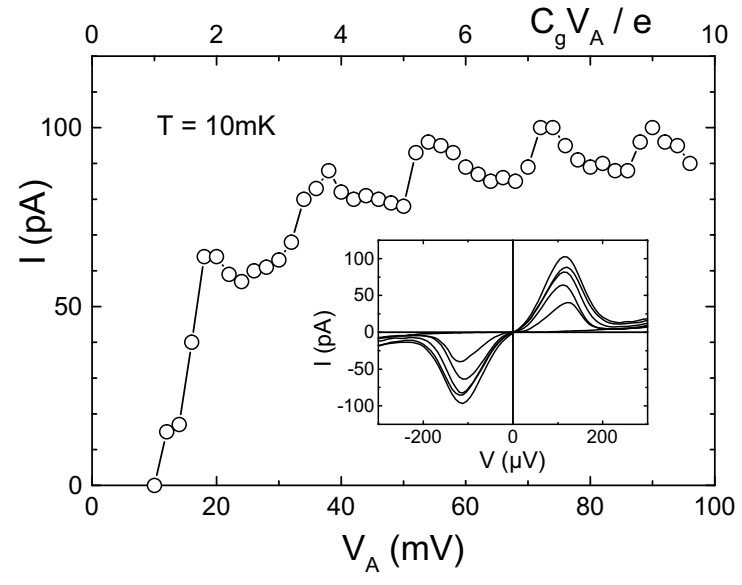

FIG. 6: Dependence of rf-induced current peak heights on the amplitude of $\mathrm{rf}$ cycling in sample 3 with significant dissipation $\left(R_{\mathrm{Cr}} \approx 12 k \Omega\right)$. The gate cycling frequency was $f=3 \mathrm{MHz}$. The cycle was centered in the middle of the blockade domain. The shapes of rf-induced current peaks for this device are presented in the inset for different amplitudes $V_{\mathrm{A}}=12,16$, 20, 40, 60, $72 \mathrm{mV}$ (in the order of peak increase). Note the significant blockade at the smallest cycling amplitude $V_{\mathrm{A}}=$ 12 caused by insufficient gate sweep and small value of CTcurrent $I_{\text {ct }} \sim 1 \mathrm{pA}$ at $V \sim 100-200 \mu \mathrm{eV}$.

ing the dependence for sample 3 with $R_{\mathrm{Cr}} \approx 12 k \Omega$ ). In our opinion, the observed quasi-periodicity on $V_{\mathrm{A}}$ is a result of the step-wise involvement of multiple open domains in gate cycling, each contributing one pulse of ST current. In good agreement with this model, every period consists of two distinct segments: sharp increase in the peak height just above the values $V_{\mathrm{A}}=(2 n+1) e / C_{\mathrm{g}}$, $n=0,1,2, \ldots$, where an additional open domain becomes involved, and gradual reduction of the height along the other part of the period, corresponding to a shortening of the duty cycle with increasing amplitudes. The inset in Fig. 6] shows the rf-induced current peaks as a function of transport voltage $V$. The position of the peaks was evidently shifted to higher voltages as compared to the sample 2, which results from the shape of the $P$-function for a relatively large dissipation in sample 3 .

\section{CONCLUSIONS}

We reported the experimental results on dissipative sequential tunneling of Cooper pairs in SETs with compact on-chip resistors. Whereas Cooper pair cotunneling was the dominant dc transport mechanism for the gate voltage range $-e / 2<C_{\mathrm{g}} V_{\mathrm{g}}<e / 2$, we were able to observe rf-induced sequential tunneling of pairs realized with the help of high-frequency gate cycling resulted in a pulsed 
ST current through the SET. We demonstrated that in accordance with theoretical predictions - the integral contribution to the net current of ST exceeds that of the $\mathrm{CT}$ in the rf-cycling mode, indicating its dominance in the vicinity of the resonant points $Q= \pm e$. By a simple model based on a quasi-static master equation approach it was possible to well describe the observed $I-V$ curve at low voltages.

Moreover, we demonstrated that, due to the effectively smaller environmental resistance seen by each single junction of an SET $\left(\sim \frac{1}{4} R_{\mathrm{Cr}}\right)$, the appreciable rf-induced ST currents can be realized even in systems with larger resistors of several $R_{\mathrm{Q}}$, where the CT current is substantially suppressed. This effect, for example, is important for possible application of discrete Cooper pair tunneling to generate accurate currents by Cooper-pair pumps [3].

Another important result of our studies is the demonstration of quasiparticle-poisoning-free performance of a superconducting SET at frequencies of $f \sim 1 \mathrm{MHz}$. This is important for the potential application of small Josephson junctions in quantum information processing, giving an estimate for a period of time of at least $\sim 1 \mu \mathrm{s}[30]$ as is available for reading out the final state of a charge qubit 31] of the loop configuration with nonzero circulating supercurrent.

\section{ACKNOWLEDGMENTS}

The work was partially supported by the EU through projects COUNT and SQUBIT-2.

* Electronic mail: Sergei.Lotkhov@ptb.de

[1] Yu. Makhlin, G. Schön, A. Schnirmann, Rev. Mod. Phys. 73, 357 (2001).

[2] A. B. Zorin, Phys. Rev. Lett. 76, 4408 (1996); A. B. Zorin, S. V. Lotkhov, Yu. A. Pashkin, H. Zangerle, V. A. Krupenin, T. Weimann, H. Scherer, and J. Niemeyer, J. Supercond. 12, 747 (1999); A. Cottet, A. Steinbach, P. Joyez, D. Vion, H. Pothier, D. Esteve and M. E. Huber, in: Macroscopic Quantum Coherence and Quantum Computing, edited by D. Averin et al. (Kluwer Academic/Plenum Publishers, New York, 2001), p. 111.

[3] L. J. Geerligs, S. M. Verbrugh, P. Hadley, J. E. Mooij, H. Pothier, P. Lafarge, C. Urbina, D. Esteve, and M. N. Devoret, Z. Phys. B: Condens. Matter 85, 349 (1991); A. B. Zorin, S. A. Bogoslovsky, S. V. Lotkhov, and J. Niemeyer, in Macroscopic Quantum Coherence and Quantum Computing, edited by D. Averin et al. (Kluwer Academic/Plenum Publishers, New York, 2001), p. 147; J. P. Pekola, J. J. Toppari, N. Kim, M. T. Savolainen, L. Taskinen, and K. Hansen, ibid, p. 127.
[4] D. V. Averin, A. B. Zorin, and K. K. Likharev, Sov. Phys. JETP 61, 407 (1985); K. K. Likharev and A. B. Zorin, J. Low Temp. Phys. 59, 347 (1985).

[5] G. L. Ingold and Yu. V. Nazarov, in Single Charge Tunneling, edited by H. Grabert and M. H. Devoret (Plenum, New York, 1992), Chap. 2.

[6] K. K. Likharev, Dynamics of Josephson Junctions and Circuits, Gordon and Breach, New York, (1986).

[7] G. L. Ingold, H. Grabert, Phys. Rev. Lett. 83, 3721 (1999); A. Steinbach, P. Joyez, A. Cottet, D. Esteve, M. H. Devoret, M. E. Huber, and John M. Martinis, Phys. Rev. Lett. 87, 137003 (2001).

[8] L. S. Kuzmin, Yu. V. Nazarov, D. B. Haviland, P. Delsing, and T. Claeson, Phys. Rev. Lett. 67, 1161 (1991).

[9] F. K. Wilhelm, G. Schön, and G. T. Zimányi, Phys. Rev. Lett. 87, 136802 (2001).

[10] J. B. Kycia, J. Chen, R. Therrien, Ç. Kurdak, K. L. Campman, A. C. Gossard, and J. Clarke, Phys. Rev. Lett. 87, 017002 (2002).

[11] W. Lu, A. J. Rimberg, and K. D. Maranowski, Appl. Phys. Lett. 81, 4976 (2002).

[12] S. V. Lotkhov, S. A. Bogoslovsky, A. B. Zorin, J. Niemeyer, Phys. Rev. Lett. 91, 197002 (2003).

[13] A. M. v. d. Brink, A. A. Odintsov, P. A. Bobbert, and G. Schön, Z. Phys. B 85, 459 (1991).

[14] D. B. Haviland, Y. Harada, P. Delsing, C. D. Chen, and T. Claeson, Phys. Rev. Lett. 73, 1541 (1994).

[15] L. J. Geerligs, V. F. Anderegg, J. Romijn, J. E. Mooij, Phys. Rev. Lett. 65, 377 (1990).

[16] M. T. Tuominen, J. M. Hergenrother, T. S. Tighe, and M. Tinkham, Phys. Rev. Lett. 69, 1997 (1992).

[17] T. A. Fulton, P. L. Gammel, D. J. Bishop, and L. N. Dunkleberger, Phys. Rev. Lett. 63, 1307 (1989).

[18] M. T. Tuominen, J. M. Hergenrother, T. S. Tighe, and M. Tinkham, IEEE Trans. Appl. Supercond. 3, 1972 (1993).

[19] A. Amar, D. Song, C. J. Lobb, and F. C. Wellstood, Phys. Rev. Lett. 72, 3234 (1994).

[20] D. V. Averin and K. K. Likharev, in: MesoscopicphenomenainSolids, edited by B. L. Altshuler, P. A. Lee, and R. A. Webb, Elsevier, Amsterdam, 173 (1991).

[21] D. V. Averin, Yu. V. Nazarov and A. A. Odintsov, Physica B 165\&166, 945 (1990).

[22] G. L. Ingold, H. Grabert, and U. Eberhardt, Phys. Rev. B 50, 395 (1994).

[23] S. V. Lotkhov, S. A. Bogoslovsky, A. B. Zorin, and J. Niemeyer, in International Workshop on Superconducting Nano-Electronics Devices, edited by J. Pekola et al. (Kluwer Academic/Plenum Publishers, New York, 2002), p. 105.

[24] A. B. Zorin, S. V. Lotkhov, H. Zangerle, and J. Niemeyer, J. Appl. Phys. 88, 2665 (2000).

[25] A. B. Zorin, Rev. Sci. Instrum. 66, 4296 (1995).

[26] D. V. Averin and Yu. V. Nazarov, Phys. Rev. Lett. 69, 1993 (1992).

[27] P. Lafarge, P. Joyez, D. Esteve, C. Urbina, and M. H. Devoret, Phys. Rev. Lett. 70, 994 (1993).

[28] P. Joyez, P. Lafarge, A. Filipe, D. Esteve, C. Urbina, and M. H. Devoret, Phys. Rev. Lett. 72, 2458 (1994).

[29] J. Männik and J. E. Lukens, report, http://arxiv.org/abs/cond-mat/0305190, (2003).

[30] In some experiments, we were able to trace gradually decreasing rf-induced currents down to frequencies of 
$\sim 1 \mathrm{kHz}$, which indicates that even longer times are, in principle, available for QPP-free operation.

[31] D. Vion, A. Aassime, A. Cottet, P. Joyez, H. Pothier,
C. Urbina, D. Esteve and M. H. Devoret, Science 296 , 886 (2002); A. B. Zorin, Physica C 368, 284 (2002). 